\title{
BMJ open Social differences in lung cancer management and survival in South East England: a cohort study
}

\author{
Anders Berglund, ${ }^{1,2}$ Mats Lambe, ${ }^{1,2}$ Margreet Lüchtenborg, ${ }^{3}$ Karen Linklater, ${ }^{3}$ \\ Michael D Peake, ${ }^{4,5}$ Lars Holmberg, ${ }^{2,3}$ Henrik Møller ${ }^{3}$
}

To cite: Berglund A, Lambe $\mathrm{M}$, Lüchtenborg $\mathrm{M}$, et al. Social differences in lung cancer management and survival in South East England: a cohort study. BMJ Open 2012;2:e001048. doi:10.1136/

bmjopen-2012-001048

- Prepublication history for this paper is available online. To view these files please visit the journal online (http:// dx.doi.org/10.1136/ bmjopen-2012-001048).

Consent was not obtained but the presented data are anonymous and the risk of identification is low.

Received 24 February 2012 Accepted 23 April 2012

This final article is available for use under the terms of the Creative Commons Attribution Non-Commercial 2.0 Licence; see http://bmjopen.bmj.com

For numbered affiliations see end of article.

Correspondence to Dr Anders Berglund; anders.berglund@ki.se

\section{ABSTRACT}

Objective: To examine possible social variations in lung cancer survival and assess if any such gradients can be attributed to social differences in comorbidity, stage at diagnosis or treatment.

Design: Population-based cohort identified in the Thames Cancer Registry.

Setting: South East England.

Participants: 15582 lung cancer patients diagnosed between 2006 and 2008.

Main outcome measures: Stage at diagnosis, surgery, radiotherapy, chemotherapy and survival.

Results: The likelihood of being diagnosed as having early-stage disease did not vary by socioeconomic quintiles $(p=0.58)$. In early-stage non-small-cell lung cancer, the likelihood of undergoing surgery was lowest in the most deprived group. There were no socioeconomic differences in the likelihood of receiving radiotherapy in stage III disease, while in advanced disease and in small-cell lung cancer, receipt of chemotherapy differed over socioeconomic quintiles $(p<0.01)$. In early-stage disease and following adjustment for confounders, the HR between the most deprived and the most affluent group was 1.24 (95\% Cl 0.98 to 1.56). Corresponding estimates in stage III and advanced disease or small-cell lung cancer were $1.16(95 \% \mathrm{Cl} 1.01$ to 1.34$)$ and $1.12(95 \% \mathrm{Cl} 1.05$ to $1.20)$, respectively. In early-stage disease, the crude $\mathrm{HR}$ between the most deprived and the most affluent group was approximately 1.4 and constant through follow-up, while in patients with advanced disease or small-cell lung cancer, no difference was detectable after 3 months.

Conclusion: We observed socioeconomic variations in management and survival in patients diagnosed as having lung cancer in South East England between 2006 and 2008, differences which could not fully be explained by social differences in stage at diagnosis, co-morbidity and treatment. The survival observed in the most affluent group should set the target for what is achievable for all lung cancer patients, managed in the same healthcare system.

\section{ARTICLE SUMMARY}

\section{Article focus}

- Social differences in management and survival in lung cancer patients.

- Particular focus on possible social variations in lung cancer survival and assess if any such gradients can be attributed to social differences in co-morbidity, stage at diagnosis or treatment.

\section{Key messages}

- There were no detectable socioeconomic differences in stage at diagnosis among lung cancer patients in South East England between 2006 and 2008.

- Socioeconomic differences in lung cancer management and survival existed. The observed inequalities in survival could not fully be explained by social differences in stage at diagnosis, co-morbidity and treatment factors.

- In early-stage disease, social gradients in survival existed throughout follow-up, whereas in advanced disease, variations in survival were confined to the period immediately after diagnosis.

Strengths and limitations of this study

- Strengths included the population-based cohort design. The material at hand allowed analyses that accounted for co-morbidity, stage at diagnosis and treatment factors.

- Limitations included the absence of data on performance status, forced expiratory volume, smoking history and lifestyle factors.

The overall prognosis of lung cancer is particularly poor in the UK with recent estimates showing a 5-year relative survival of $6.5 \%$ and $8.4 \%$ for men and women, respectively. ${ }^{1}$ Observed national differences appear to be most pronounced early in the period of follow-up. ${ }^{2}$ Outcome in earlystage lung cancer is highly dependent on management, where pulmonary resection offers a potentially curative treatment modality. ${ }^{3}{ }^{4}$ Approximately $10 \%$ of all lung cancer patients in the UK underwent surgical 
resection between 2004 and $2006,{ }^{5}$ which is lower than corresponding estimates in some other European countries and the USA. ${ }^{6}$ Previous studies have found evidence of both regional variations in treatment intensity and socioeconomic inequalities in lung cancer survival in the UK. ${ }^{8-12}$ While one important aim of the National Health Service Cancer Plan in 2000 was to reduce social inequalities in cancer survival, ${ }^{13}$ marked differences persisted 10 years later. ${ }^{14}$ Several factors may contribute to social gradients in cancer survival including the patient's general health status, knowledge and healthcare seeking behaviours, characteristics of the tumour at time of diagnosis and clinical management. Differences in the management of patients in relation to socioeconomic factors, including the use of surgery in non-small-cell lung cancer patients, have previously been reported in the UK and in other countries. ${ }^{15} 16$ The present study was based on patients diagnosed as having lung cancer identified in the Thames Cancer Registry between 2006 and 2008 and aimed to examine possible social gradients in lung cancer survival and assess if any such variation can be attributed to social differences in co-morbidity, stage at diagnosis and treatment.

\section{METHODS}

\section{Data collection}

The Thames Cancer Registry is population based and currently covers a population of 12 million people in South East England (London, Kent, Surrey and Sussex). The Thames Cancer Registry registers approximately 57000 incident cancer cases annually. Cancer registration is based on clinical and pathological information received from hospitals and from death certificates provided by the Office for National Statistics. Hospital episode statistics data are obtained from hospital patient administration systems and include information on the patient, as well as clinical and administrative details. The UK National Lung Cancer Audit was set up in 2004 as a clinical database for all new lung cancer patients. The number of trusts participating in this audit increased from $40 \%$ in 2005 to $85 \%$ in England and Wales in 2008. ${ }^{17}$ While information on items such as stage at diagnosis and treatment details are incompletely recorded in the cancer registry data set, these can be supplemented by means of record linkage to the National Lung Cancer Audit and hospital episode statistics. ${ }^{18}$ The large majority of the relevant healthcare providers in South East England are part of a national tax-funded health system, with only a minority of lung cancer patients being managed by private providers.

For the purpose of the present study, we identified lung cancer cases registered with International Classification of Diseases-10th Revision codes C33 and C34 from the Thames Cancer Registry database. Data on surgery and radiotherapy were retrieved from hospital episode statistics and supplemented with data from Thames Cancer Registry, while information on stage and chemotherapy was obtained from the Cancer Registry and supplemented with data from the National Lung Cancer Audit. Information on stage at diagnosis was available for $72.7 \%$ of the patients. The definition of primary initial treatment (surgical resection, radiotherapy and chemotherapy) was based on simple binary indicators. No treatment and no record of treatment cannot be distinguished in these data.

\section{Study population}

A total of 16183 patients with a diagnosis of lung cancer between 2006 and 2008 were identified in the Thames Cancer Registry. Following exclusion of death certificateonly cancer registrations (601 cases or $3.7 \%$ of the total), the final study population consisted of 15582 cases. Patients were subdivided into three subgroups according to disease status: early-stage (stages IA-IIB) non-smallcell lung cancer $(n=1828)$, stage III non-small or smallcell lung cancer $(n=2771)$ and advanced stage (stages IIIA-IV) disease or all stages of small-cell lung cancer $(n=10039)$. These three groups were created on the basis of the most commonly used initial treatment modalities in every subgroup.

\section{Co-morbidity}

A modified version of the Charlson comorbidity index was used to assess the burden of concomitant disease for each patient, following retrieval of information on comorbidity from the hospital episode statistics in a 3-year period preceding the lung cancer diagnosis. The original Charlson comorbidity index consists of 19 disease groups and has been used in earlier studies that have assessed the role of concomitant disease in cancer patients. ${ }^{19-21}$ In the present study, all groups except cancer were included. Each disease group was then assigned a specific weight (1, 2, 3 and 6) depending on the severity of the medical condition. The Charlson index was derived by summing the weighted scores for all comorbidities. On the basis of the distribution of the Charlson score, the patients were classified into three comorbidity categories: no (0), mild (1) and severe comorbidity $(2+)$. Information was available for $88.4 \%$ of the patients. For 1808 patients, no link to the hospital episode statistics data could be established.

\section{Socioeconomic quintile}

Socioeconomic status recorded in the Thames Cancer Registry is based on the income domain of the 2007 Indices of Deprivation $^{22}$ in which patients are classified based on their postcode of residence and are categorised into quintiles of socioeconomic deprivation. The most affluent group was labelled Q1 and the most deprived Q5.

\section{Statistical methods}

Among all patients, crude and adjusted binary logistic regression models with ORs and $95 \%$ CIs were used for prediction of the probability of being diagnosed as having early-stage disease by socioeconomic quintile. In a subsequent step, the likelihood to undergo surgical resection (in early-stage non-small-cell lung cancer) to receive 


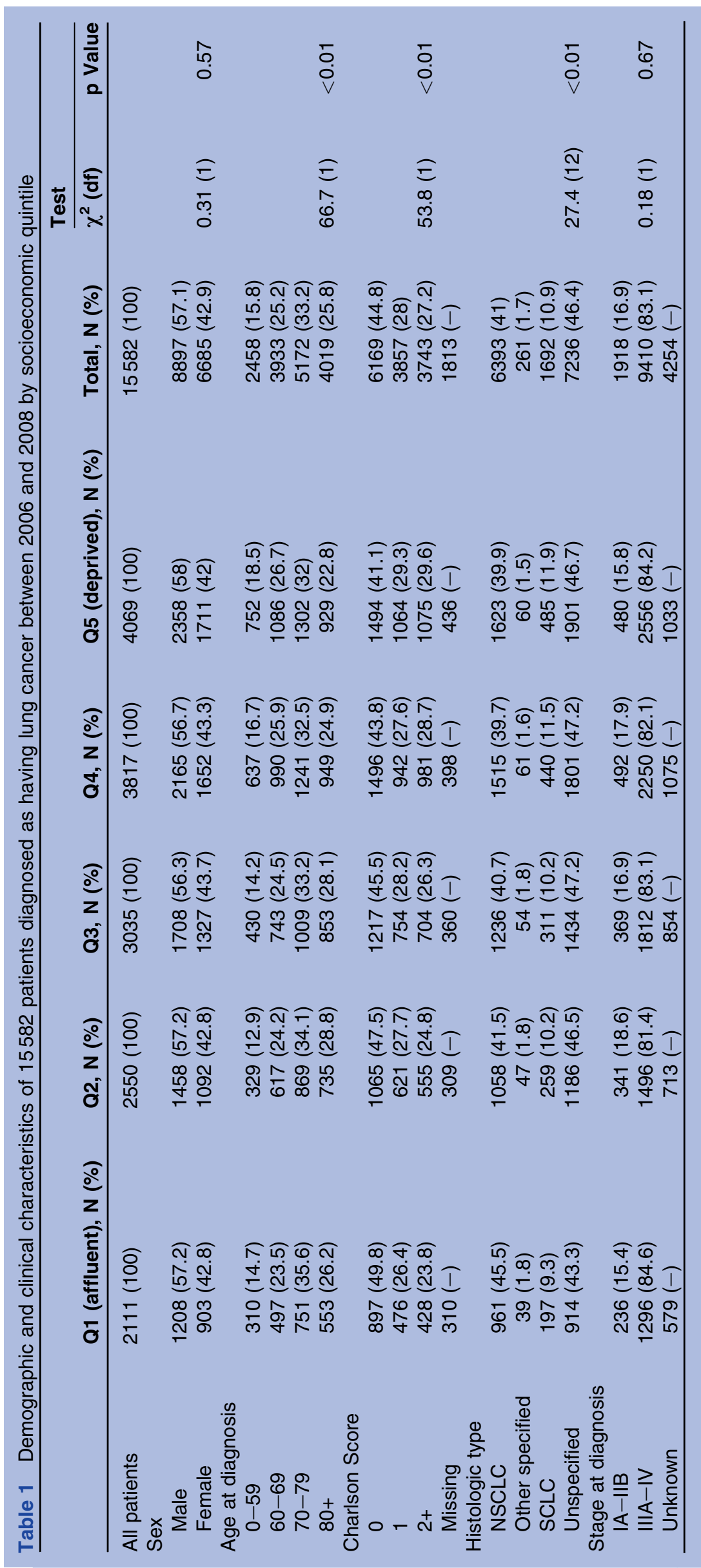


radiotherapy (in stage III disease) and to receive chemotherapy (in advanced disease or small-cell lung cancer) were assessed by using crude and adjusted binary logistic regression models. All models were adjusted for sex, age at diagnosis and the Charlson comorbidity index.

In time to event analysis, the outcome of interest was all-cause mortality. Survival time was defined as the interval between the date of the lung cancer diagnosis and the date of death, emigration or end of follow-up on 31 December 2009. For each clinical subgroup (earlystage non-small-cell lung cancer, stage III disease and advanced stage or small-cell lung cancer), Cox regression models were used to calculate HRs and 95\% CIs for all-cause mortality in relation to socioeconomic quintiles. Both univariate and multivariate models adjusted for sex, age at diagnosis, co-morbidity and treatment factors were used. We used multiple imputation to account for missing information on co-morbidity using chained equations with 60 imputated data sets. ${ }^{23}$

The relative contribution (\%) of adding each covariate separately in explaining the possible social variation in mortality between the most affluent and the most deprived patients in each subgroup was calculated as follows: ((HR for $\mathrm{SES}_{\mathrm{Q} 5}$ in model A - HR for $\mathrm{SES}_{\mathrm{Q} 5}$ in model B) $/\left(\mathrm{HR}\right.$ for $\mathrm{SES}_{\mathrm{Q} 5}$ in model A - 1) $) \times 100$, where model A is the basic model (socioeconomic quintiles and adjusted for sex) and in model B, with each covariate being added to model $\mathrm{A}$.

Cumulative survival and mortality rates (per 100 personyears) were estimated by subgroups and socioeconomic quintile. The mortality rates were calculated as number of deaths divided by the person-years at risk. These estimates were modelled with flexible parametric survival models using a restricted cubic spline for the baseline mortality rate. ${ }^{24}$ These models, similar to Cox regression models, provide HRs as measures of association between exposures and outcome. By modelling the underlying rate parametrically, it is possible to estimate fitted curves from the model, such as the cumulative survival function. In the Cox regression models, we estimated a single HR between socioeconomic quintiles, assumed to be constant throughout follow-up. In the flexible parametric survival framework, the HR between the most deprived versus most affluent patients was estimated in predefined segments of the follow-up, using a second spline function. The HR is then time dependent on the underlying timescale that was time since diagnosis of lung cancer. All flexible parametric survival models were estimated using the stpm2-package in STATA. ${ }^{25}$

In all analyses, we computed $\chi^{2}$ tests and $p$ values for trend by fitting a linear categorical variable. All $p$ values are two sided, and statistical significance was considered at $\mathrm{p}<0.05$. The statistical analyses were performed using STATA V.11, and graphical displays were produced using R V. $10.2 \cdot{ }^{26}$

\section{RESULTS}

\section{Demographic and clinical characteristics}

Table 1 shows the demographic and clinical characteristics by socioeconomic quintile. The most affluent

Table 2 The likelihood of being diagnosed as having early-stage disease* estimated by crude and adjusted regression models with ORs and $95 \% \mathrm{Cls}$

\begin{tabular}{|c|c|c|c|c|c|}
\hline & \multirow[b]{2}{*}{$\mathbf{N}(\%)$} & \multicolumn{2}{|l|}{ Crude } & \multicolumn{2}{|c|}{ Adjusted } \\
\hline & & OR & $95 \% \mathrm{Cl}$ & OR & $95 \% \mathrm{Cl}$ \\
\hline \multicolumn{6}{|l|}{ Socioeconomic quintile } \\
\hline Q1 (affluent) & $236(15.4)$ & 1.00 & Reference & 1.00 & Reference \\
\hline Q2 & $341(18.6)$ & 1.25 & 1.04 to 1.50 & 1.24 & 1.04 to 1.49 \\
\hline Q3 & 369 (16.9) & 1.12 & 0.93 to 1.34 & 1.11 & 0.93 to 1.33 \\
\hline Q4 & $492(17.9)$ & 1.20 & 1.01 to 1.42 & 1.18 & 1.00 to 1.40 \\
\hline Q5 (deprived) & $480(15.8)$ & 1.03 & 0.87 to 1.22 & 1.01 & 0.85 to 1.20 \\
\hline Test for trend ( $\chi^{2}, p$ value $)$ & & 0.18 & 0.67 & 0.59 & 0.44 \\
\hline \multicolumn{6}{|l|}{ Sex } \\
\hline Male & 1037 (15.9) & 1.00 & Reference & 1.00 & Reference \\
\hline Female & 881 (18.3) & 1.19 & 1.08 to 1.30 & 1.10 & 1.10 to 1.33 \\
\hline Test for trend ( $\chi^{2}, p$ value $)$ & & 11.5 & $<0.01$ & 12.6 & $<0.01$ \\
\hline \multicolumn{6}{|l|}{ Age at diagnosis } \\
\hline $0-59$ & $300(15.2)$ & 1.00 & Reference & 1.00 & Reference \\
\hline $60-69$ & $550(17.6)$ & 1.19 & 1.02 to 1.39 & 1.16 & 0.99 to 1.35 \\
\hline $70-79$ & $694(18.4)$ & 1.26 & 1.08 to 1.46 & 1.18 & 1.01 to 1.37 \\
\hline $80+$ & $374(15.3)$ & 1.01 & 0.85 to 1.19 & 0.93 & 0.78 to 1.10 \\
\hline Test for trend ( $\chi^{2}, p$ value $)$ & & 2.75 & 0.10 & 0.29 & 0.59 \\
\hline \multicolumn{6}{|l|}{ Charlson Score } \\
\hline 0 & $719(15.0)$ & 1.00 & Reference & 1.00 & Reference \\
\hline 1 & $511(17.6)$ & 1.19 & 1.05 to 1.34 & 1.19 & 1.05 to 1.34 \\
\hline $2+$ & $504(19.0)$ & 1.29 & 1.14 to 1.47 & 1.31 & 1.15 to 1.49 \\
\hline Test for trend ( $\chi^{2}, p$ value $)$ & & 20.0 & $<0.01$ & 21.7 & $<0.01$ \\
\hline
\end{tabular}




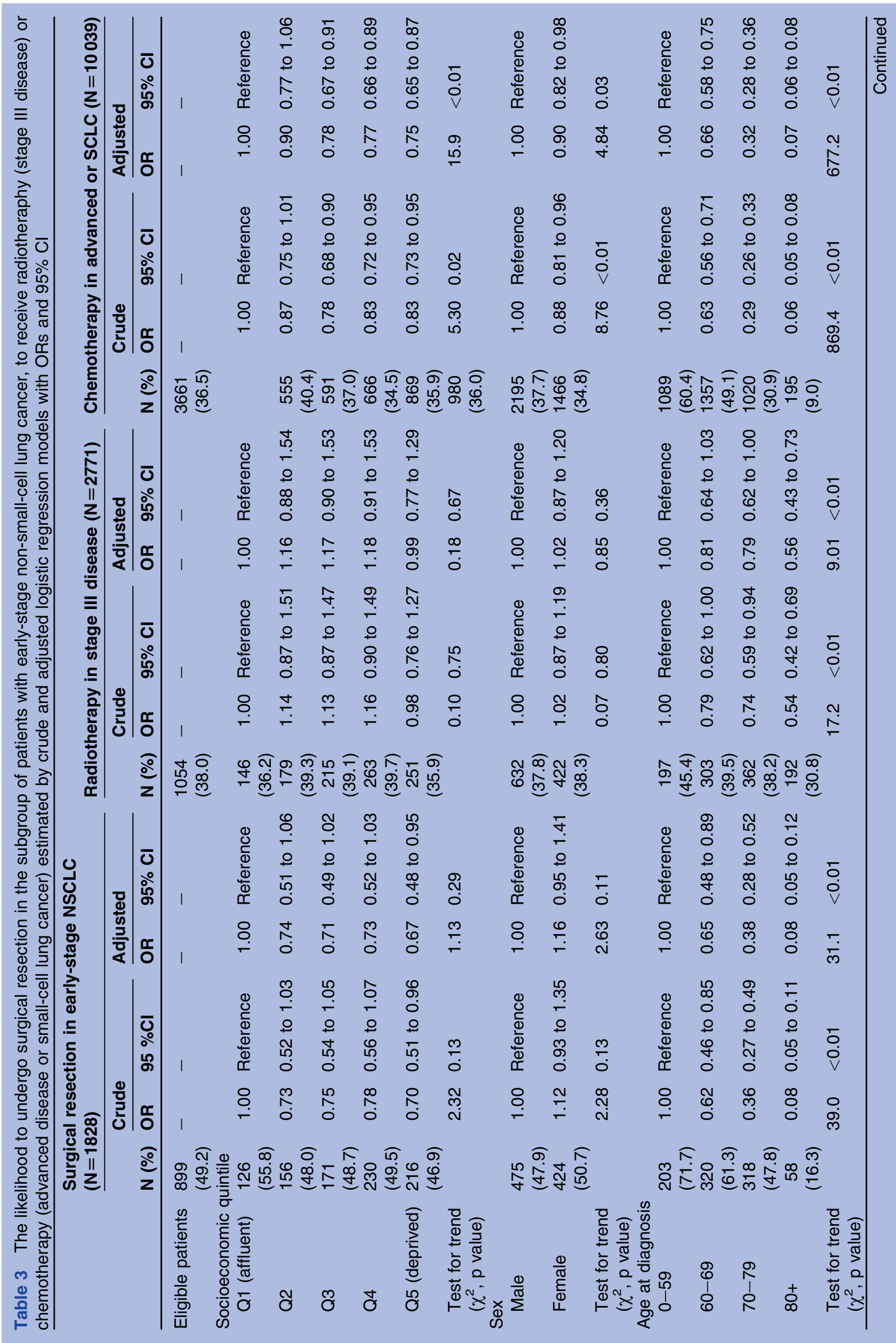




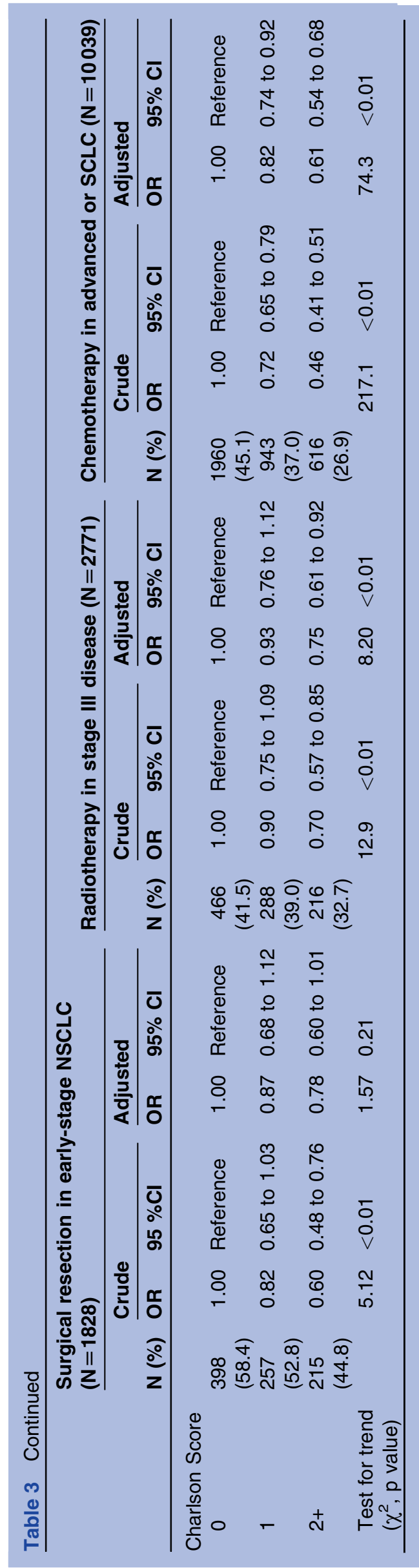

patients were generally older than the most deprived patients $(\mathrm{p}<0.01)$. The proportion of patients with severe co-morbidity was higher in people categorised as deprived $(29.6 \%)$ in Q5 than in people belonging to the affluent $(23.8 \%)$ in Q1 $(\mathrm{p}<0.01)$. Among patients with classifiable disease, the proportion of non-small-cell lung cancer was higher in the most affluent group. No statistically significant trend over the socioeconomic quintiles was observed with regard to the likelihood to be diagnosed as having early-stage disease $(\mathrm{p}=0.44)$ (table $2)$. However, patients with co-morbidity were more likely to be diagnosed as having early-stage disease $(\mathrm{p}<0.01)$.

\section{Treatment modality}

About half of patients (49.2\%) with early-stage nonsmall-cell lung cancer underwent surgical resection. Surgery decreased from $55.8 \%$ in the most affluent group to $46.9 \%$ in the most deprived group $(p=0.29)$ (table 3). There was a significant reduction in surgical resection with increasing age at diagnosis (trend $\mathrm{p}<0.01$ ), but not in relation to co-morbidity (trend $p=0.21$ ), once other variables were adjusted for. In patients with stage III disease, $1054(38.0 \%)$ had radiotherapy treatment recorded (table 3). In this subgroup, there was no association between socioeconomic quintile and receipt of radiotherapy $(\mathrm{p}=0.67)$. In 10039 patients registered with advanced disease or small-cell lung cancer, $36.5 \%$ had a record of chemotherapy (table 3). A statistically significant trend of reduced odds to receive chemotherapy was observed with increasing levels of socioeconomic deprivation $(p<0.01)$ and with increased age at diagnosis $(\mathrm{p}<0.01)$ and co-morbidity burden $(\mathrm{p}<0.01)$.

\section{Survival}

Figure 1 shows estimated cumulative survival functions by socioeconomic quintile generated by flexible parametric models. In patients with early-stage non-small-cell lung cancer, the 3-year survival in the most affluent and the most deprived group was $50 \%$ and $39 \%$, respectively. While survival in stage III disease and in advanced disease or small-cell lung cancer was poor in all socioeconomic quintiles, the prognosis was somewhat better in the most affluent group.

Table 4 shows the risk of all-cause mortality in patients with early-stage non-small-cell lung cancer, in stage III disease and advanced disease or small-cell lung cancer. In early-stage non-small-cell lung cancer, the HR between the most deprived versus most affluent group was 1.24 (95\% CI 0.98 to 1.56) following adjustment for potential confounders. The corresponding estimates in stage III disease and advanced disease or small-cell lung cancer was 1.16 (95\% CI 1.01 to 1.34 ) and 1.12 (95\% CI 1.05 to 1.20$)$, respectively. In none of the three clinical subgroups, no independent statistically significant trend by socioeconomic quintiles could be detected.

The relative contribution of age at diagnosis explaining the social inequality in survival between the most affluent and the most deprived patients was 34.6\% in stage III disease and $13.9 \%$ in advanced disease or 
A

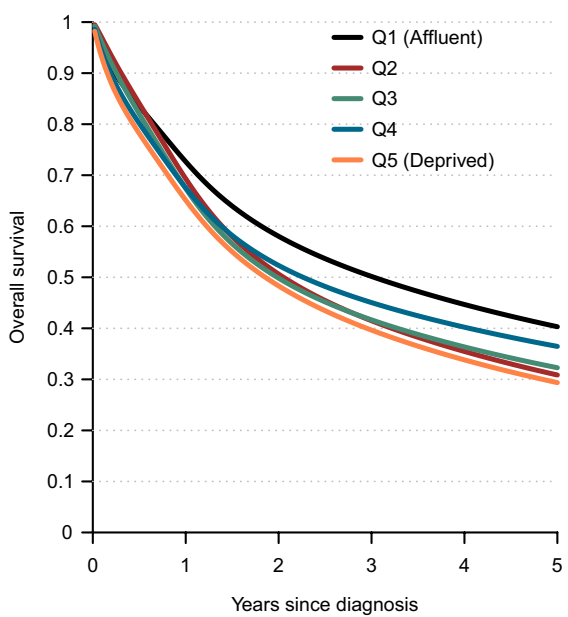

B

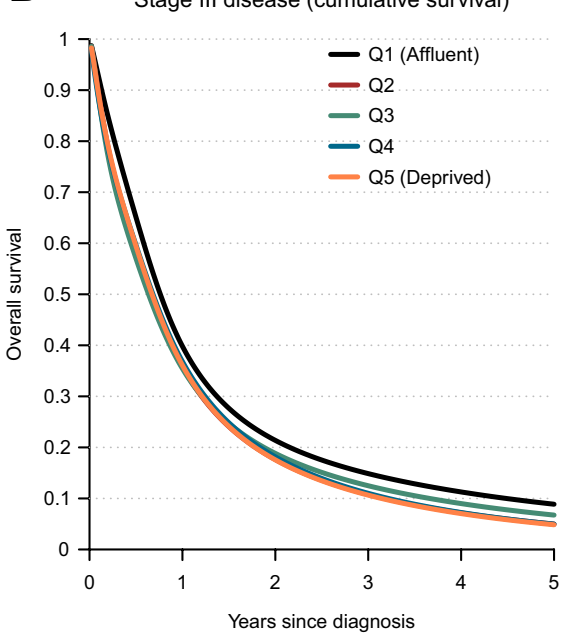

C

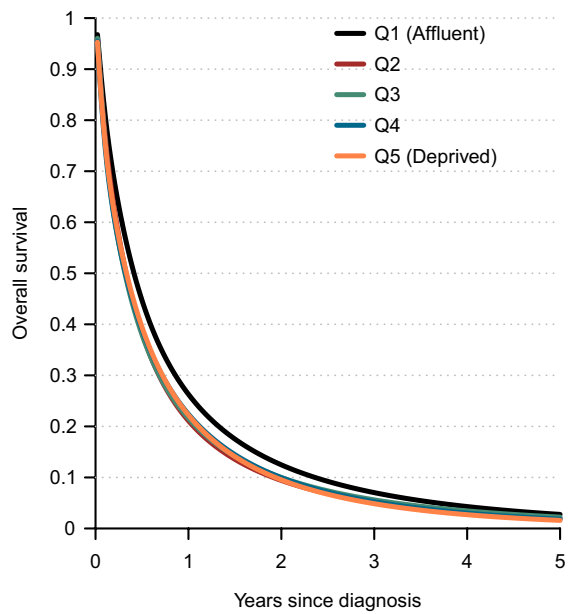

Figure 1 Cumulative survival estimated by flexible parametric models by tumour subgroups and socioeconomic quintile. (A) Early-stage non-small-cell lung cancer (NSCLC). (B) Stage III disease. (C) Advanced disease or small-cell lung cancer (SCLC).

small-cell lung cancer (table 4). The corresponding percentages of co-morbidity burden explaining the social inequality between the most affluent and the most deprived patients was $17.4 \%$ in stage III disease and $11.0 \%$ in advanced disease or small-cell lung cancer. Resection explained $42.3 \%$ in early-stage disease and chemotherapy explained $23.4 \%$ in advanced disease or small-cell lung cancer of the social variation in survival.

Figure 2 illustrates the variation in overall mortality rates by time since diagnosis in the five socioeconomic quintiles and the variation of HRs between the most deprived and the most affluent group. In early-stage non-small-cell lung cancer, socioeconomic variations in the mortality rates were most pronounced in the first months after diagnosis, but the differences persisted 12 months after diagnosis. A HR of approximately 1.4 between the most deprived and the most affluent group remained constant during the follow-up.

In stage III disease, and in advanced disease or smallcell lung cancer, the mortality rates peaked and varied by socioeconomic quintiles primarily during the first month postdiagnosis, but after 12 months, only a minor social difference was observed. The same pattern was observed in the time-dependent HR between the most deprived and the most affluent group.

\section{DISCUSSION}

\section{Main findings}

Our study clearly demonstrates the presence of social differences in management, and in survival in patients diagnosed as having lung cancer in South East England between 2006 and 2008, and observed social differences could not fully be explained by differences in stage at diagnosis, co-morbidity and treatment factors. In earlystage disease, the social gradients existed throughout the follow-up, whereas in advanced disease variations in survival were confined to the period immediately after diagnosis.

\section{Methodological strengths and limitations}

Strengths of our study included the population-based approach where the analyses were based on a cohort that included virtually all lung cancer patients diagnosed and managed in South East England between 2006 and 2008. For the purpose of the present study, additional information was obtained from other sources than the Thames Cancer Registry, including information on stage at diagnosis and co-morbidity. A weakness was the absence of stage at diagnosis for one-fourth of the patients, which varied from $25.4 \%$ (Q5) to 27.9\% (Q1) by socioeconomic quintiles. Patients with missing information on stage at diagnosis had particularly poor prognosis, which indicates that they often had advanced disease. Co-morbidity burden was assessed by using the Charlson comorbidity index that previously has been shown to be associated with the management of and outcomes in cancer patients, ${ }^{20}$ including lung cancer. ${ }^{27}$ However, since no information was available on medical conditions not requiring inhospital care and on other types of malignancies, it cannot be excluded that the influence of concomitant disease was underestimated. The introduction of the 7 th edition of the tumour, node, metastases classification in 2009 provides better prognostic differentiation. ${ }^{28}$ Since our study included cases diagnosed between 2006 and 2008, some caution should be taken when a reader generalise the findings to the situation of today. Also, the data at hand did not allow for assessment of performance status, forced expiratory volume, smoking history and life style factors. Thus, concomitant disease, poor performance status and impaired lung function may have been both more common and more severe in patients with low socioeconomic status, ultimately influencing choice of treatment.

The definition of primary treatment (surgical resection, radiotherapy and chemotherapy) was based on the registration of 'Yes' or 'Unknown'. Thus, it was unclear if 


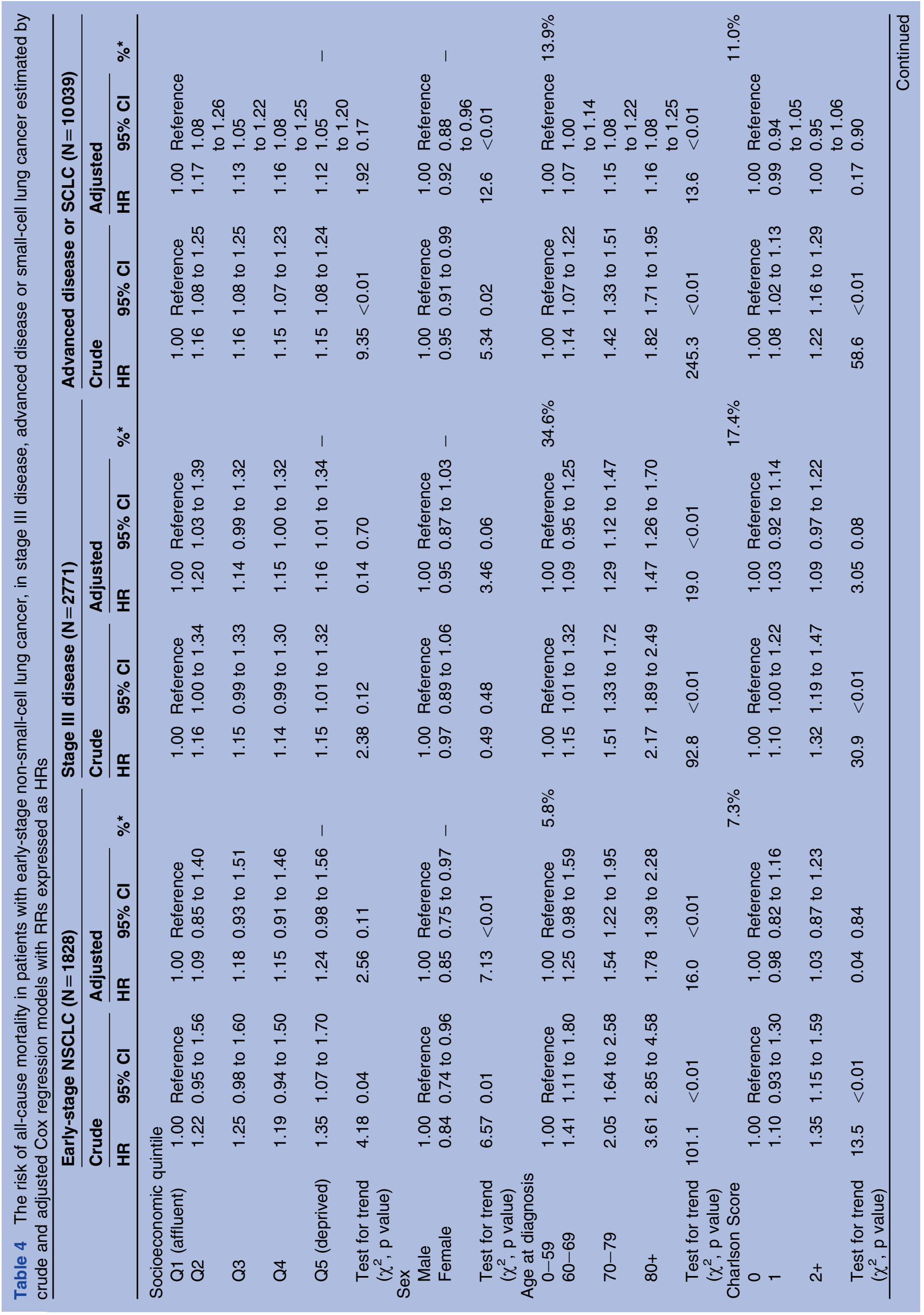




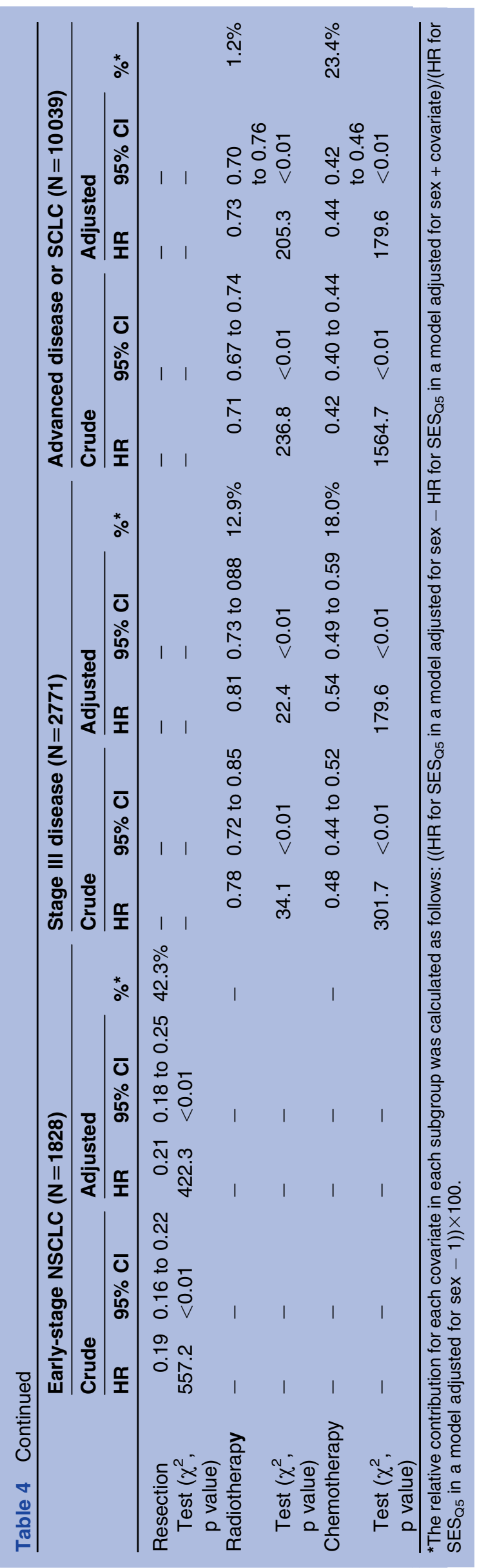

'Unknown' was registered as meaning no treatment or missing information. However, it appears unlikely that there would be a systematic misclassification of treatment modality, favouring the most affluent patients. We acknowledge that our study included no information on second-line treatments or smoking history. Although the income domain of the Indices of Deprivation used in the present study is an established indicator of socioeconomic status, it might not cover all aspects that contribute to the meaning of social status. Using an areabased deprivation index is likely to lead to an underestimation of social differences in survival. ${ }^{29}$ The variability in socioeconomic status based on area characteristics will always be smaller than that on the individual level. ${ }^{30}$ In analyses without multiple imputation for missing values on co-morbidity, results remained virtually unchanged with regard to social gradients in management and survival. Finally, the interpretation of results in some subgroups (eg, early-stage disease) may have been hampered by a small number of events.

\section{Comparison with other studies}

Patients diagnosed as having lung cancer are predominantly elderly smokers or ex-smokers who often have other smoking-associated illnesses. We observed social variations in co-morbidity burden, but there were no differences with regard to stage at diagnosis between socioeconomic quintiles, a finding that corroborates with results from earlier studies. ${ }^{16} 31$ However, a recent population-based Danish study found associations between level of education and stage at diagnosis, ${ }^{32}$ which is in line with results from a study from USA. ${ }^{33}$ In the present study, early-stage disease was more common in patients with severe co-morbidity, which may reflect that the lung malignancy was detected in the course of medical attention for other conditions.

In the present as well as in earlier studies conducted in Europe, choice of lung cancer management appears to vary by social group. ${ }^{16}{ }^{34}$ Corroborating results from earlier studies, ${ }^{15} 16$ we observed social gradients in the likelihood to undergo surgery, a finding that was independent of age at diagnosis and co-morbidity burden. The majority of lung cancer patients are diagnosed as having advanced disease, where the primary goal of treatment is to control the cancer and alleviate symptoms, such as cough or breathlessness. Recommended initial treatments in advanced disease include radiotherapy and/or chemotherapy. The 2005 Guidelines on the management of lung cancer from the National Institute for Health and Clinical Excellence and updated in 2011 recommend chemotherapy in small-cell lung cancer, given that patients are fit enough. ${ }^{3}$ In line with our findings, socioeconomic factors have previously been shown to be associated with chemotherapy use in lung cancer in South East England. ${ }^{35}$

Social variations in management may be a result of several factors. In addition to possible differences between deprivation groups in general health status and healthcare-seeking behaviours, there may be subtle 

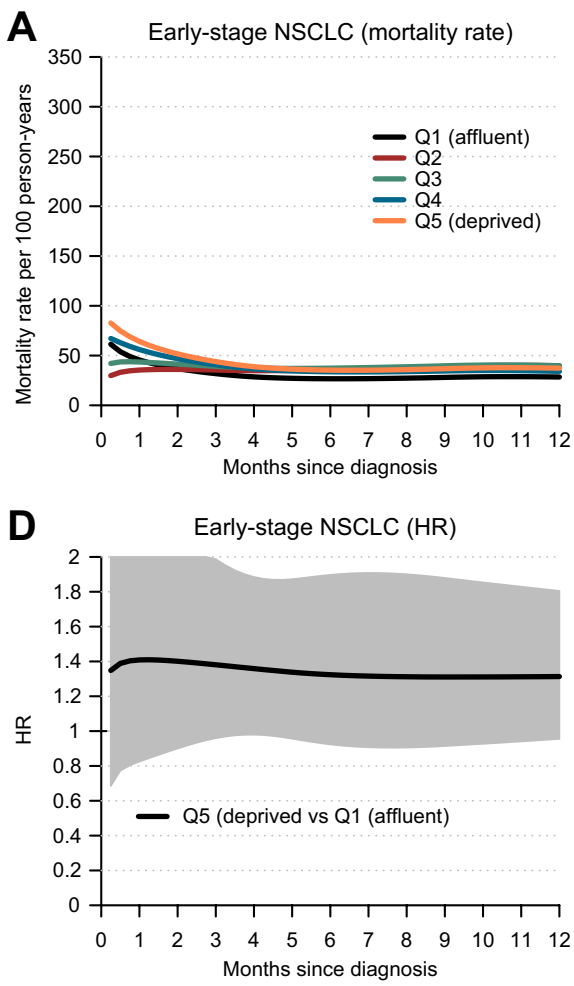

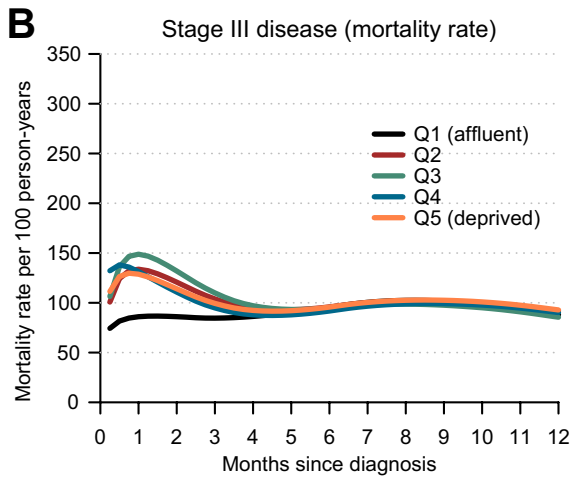

$\mathbf{E}$

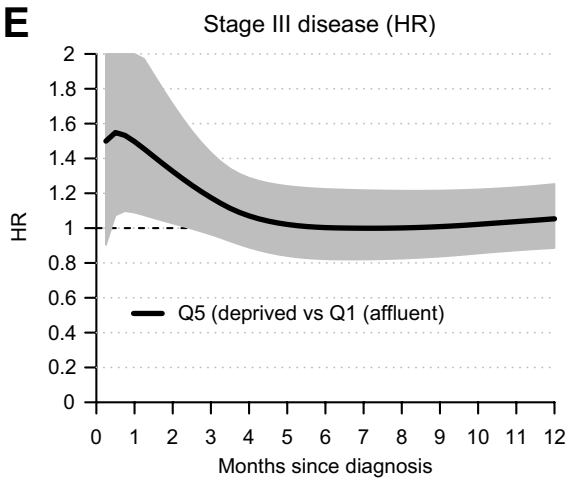

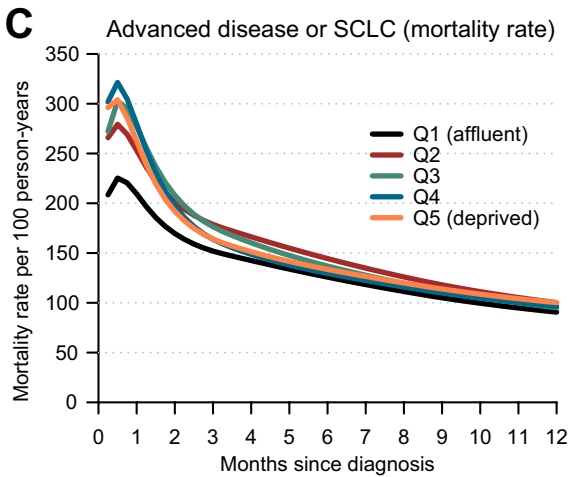

$\mathbf{F}$

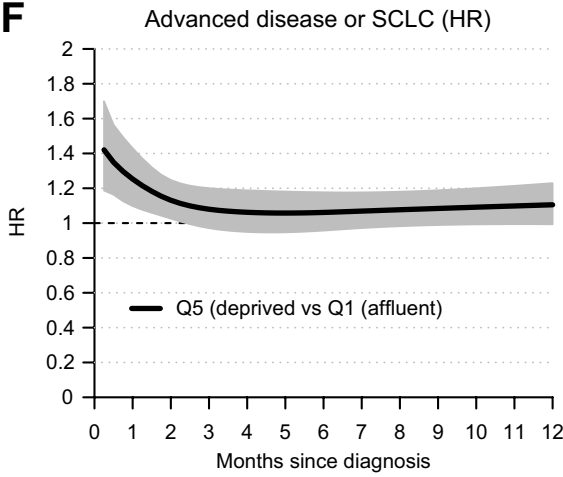

Figure 2 Estimated mortality rates by socioeconomic quintile (allowing socioeconomic quintile to vary by follow-up) and the HR between the most deprived versus the most affluent patients within 12 months of diagnosis by tumour subgroups using flexible parametric models. (A) Early-stage non-small-cell lung cancer (NSCLC) (mortality rate). (B) Stage III disease (mortality rate). (C) Advanced disease or small-cell lung cancer (SCLC) (mortality rate). (D) Early-stage NSCLC (HR). (E) Stage III disease (HR). (F) Advanced disease or SCLC (HR).

differences in the doctor-patient interaction related to socioeconomic factors. A recent Danish study found evidence of associations between educational level and time between referral and diagnosis, ${ }^{32}$ findings that corroborate results from Sweden. ${ }^{16}$ Also, more affluent patients may have better support and knowledge resulting in increased expectations and demands on the health provider. Furthermore, patients' own willingness to accept treatment and acceptance of risks associated with surgical resection may differ by level of deprivation. Finally, socioeconomic status has been associated with access to cancer services, with less affluent patients having poorer access to specialist services and treatment modalities, referred to in the UK as a 'postcode lottery' ${ }^{36}$ A recent study concluded that living in a deprived locality in northern England reduced the likelihood of undergoing treatment for lung cancer and suggested that centralisation of services (thus increasing travelling distances to specialist centres) would lead to further disadvantages for these patients, and the same phenomenon was observed in respect of access through a local hospital to any treatment and to histological diagnosis. ${ }^{37}$

Our findings of social gradients in lung cancer survival corroborate results from several earlier studies. ${ }^{8-10} 16$ Also, a recent report has documented persisting socioeconomic inequalities in cancer survival in England after the introduction of the National Health Service Cancer Plan. ${ }^{14}$ While social differences in tumour stage at time of diagnosis have often been put forward as an explanation for social inequalities in cancer survival, ${ }^{38}$ both the present and recent results from Sweden do not support this notion. ${ }^{16}$ One English study has even suggested that deprived lung cancer patients are more likely to be diagnosed as having localised disease. ${ }^{39}$ However, possible social differences in diagnostic intensity, such as a higher likelihood to be examined by Positron Emission Tomography and Computed Tomography or CT-scan in affluent groups, could have resulted in stage migration ('Will Rogers phenomenon'), ${ }^{40}$ which could inflate observed findings of social variations in survival. We have previously observed a higher diagnostic intensity in lung cancer patients in Sweden with high socioeconomic status, ${ }^{16}$ which may result in more accurate staging. Host factors that may contribute to social differences in survival include co-morbidity and lifestyle factors, both prediagnosis and postdiagnosis. In the present study, adjustment for concomitant disease contributed only marginally to the difference in the survival estimates between social groups. While no information was available on lifestyle, it cannot be excluded that the observed inequalities in survival in early-stage disease throughout follow-up may reflect social differences in smoking cessation following diagnosis. Our findings indicate that the relative contribution of differences in treatment to social inequalities in survival appears to be high foremost in early-stage 
disease. This may play a particularly important role in explaining social differences in short-term mortality rates. Thus, increased treatment intensity among deprived patients is likely to help reduce social inequalities in survival.

\section{Conclusion and policy implications}

We observed socioeconomic differences in the clinical management and in survival in patients diagnosed as having lung cancer in the South East England between 2006 and 2008. While efforts to eliminate social differences in lung cancer incidence will take many years, concerted action could reduce socioeconomic inequalities in management and survival more quickly. Areas of importance include efforts to guarantee equal access to health services and monitoring of adherence to guidelines. The survival observed in the most affluent patient group should set the target for what is achievable in the short term for all lung cancer patients managed in the same healthcare system.

\section{Author affiliations \\ ${ }^{1}$ Department of Medical Epidemiology and Biostatistics, Karolinska Institutet, Stockholm, Sweden \\ ${ }^{2}$ Regional Cancer Centre of Central Sweden, University Hospital, Uppsala, Sweden \\ ${ }^{3}$ King's College London, School of Medicine, Division of Cancer Studies, Thames Cancer Registry, London, UK \\ ${ }^{4}$ Department of Respiratory Medicine, Glenfield Hospital, Leicester, UK \\ ${ }^{5}$ National Cancer Intelligence Network, London, UK}

Contributors HM, LH, ML and AB designed and collected the data of the study. $A B$ did the statistical analyses. All authors participated in interpretation of the data. $A B$ wrote the first draft of the paper. All authors had full access to all the data and approved the final draft. HM is the guarantor for the study.

Funding $A B$ was supported by a postgraduate scholarship from the Health Care Sciences Postgraduate School at Karolinska Institutet in Sweden. The funders of the study had no role in the design and conduct of the study; collection, management, analyses and interpretation of the results and preparation, review or approval of the manuscript. The funders did not have any involvement in the design of the study, the collection, analysis and interpretation of the data; the writing of the manuscript or the decision to submit the manuscript for publication.

Competing interests None.

Ethical approval The study was approved by the Research Ethics Committee at Karolinska Institute.

Provenance and peer review Not commissioned; externally peer reviewed.

Data sharing statement No additional data are available.

\section{REFERENCES}

1. Holmberg L, Sandin F, Bray F, et al. National comparisons of lung cancer survival in England, Norway and Sweden 2001-2004: differences occur early in follow-up. Thorax 2010;65:436-41.

2. Coleman MP, Forman D, Bryant $\mathrm{H}$, et al. Cancer survival in Australia, Canada, Denmark, Norway, Sweden, and the UK, 1995-2007 (the International Cancer Benchmarking Partnership): an analysis of population-based cancer registry data. Lancet 2011;377:127-38.

3. Baldwin DR, White B, Schmidt-Hansen M, et al. Diagnosis and treatment of lung cancer: summary of updated NICE guidance. BMJ 2011;342:d2110.

4. Pearson FG. Current status of surgical resection for lung cancer. Chest 1994;106:337S-9S.
5. National Cancer Intelligence Network. NHS Treated Cancer Patients Receiving Major Surgical Resections - NCIN Briefing. London: National Cancer Intelligence Network Coordinating Centre, 2010.

6. Fry WA, Phillips JL, Menck HR. Ten-year survey of lung cancer treatment and survival in hospitals in the United States: a national cancer data base report. Cancer 1999;86:1867-76.

7. Myrdal G, Lamberg K, Lambe M, et al. Regional differences in treatment and outcome in non-small cell lung cancer: a populationbased study (Sweden). Lung Cancer 2009;63:16-22.

8. Riaz SP, Horton M, Kang J, et al. Lung cancer incidence and survival in England: an analysis by socioeconomic deprivation and urbanization. J Thorac Oncol 2011;1:1

9. Jack $\mathrm{RH}$, Davies $\mathrm{EA}$, Moller $\mathrm{H}$. Lung cancer incidence and survival in different ethnic groups in South East England. Br J Cancer 2011;105:1049-53.

10. Coleman MP, Rachet B, Woods LM, et al. Trends and socioeconomic inequalities in cancer survival in England and Wales up to 2001. Br J Cancer 2004;90:1367-73.

11. Jack RH, Gulliford MC, Ferguson J, et al. Geographical inequalities in lung cancer management and survival in South East England: evidence of variation in access to oncology services? $\mathrm{Br} \mathrm{J}$ Cancer 2003;88:1025-31.

12. NHS Information Centre. The National Lung Cancer Audit: 2011 Report. http://www.ic.nhs.uk/webfiles/Services/NCASP/audits\% 20and\%20reports/NHS_IC_Lung_Cancer_AUDIT_2011_Interactive_ PDF_V1.0.pdf (accessed 15 Jan 2012).

13. Department of Health. The NHS Cancer Plan: A Plan for Investment A Plan for Reform. London, Department of Health, 2000.

14. Rachet B, Ellis L, Maringe C, et al. Socioeconomic inequalities in cancer survival in England after the NHS cancer plan. $\mathrm{Br} J$ Cancer 2010;103:446-53.

15. McMahon M, Barbiere JM, Greenberg DC, et al. Population-based trends in use of surgery for non-small cell lung cancer in a UK region, 1995-2006. Thorax 2011;66:453-5.

16. Berglund A, Holmberg L, Tishelman C, et al. Social inequalities in non-small cell lung cancer management and survival: a population-based study in central Sweden. Thorax 2010;65:327-33.

17. The NHS Information Centre. National Lung Cancer Audit 2010. Leeds: The NHS Information Centre for Health and Social Care, 2009.

18. National Cancer Intelligence Network. National Cancer Data Repository. http://www.ncin.org.uk/collecting_and_using_data/ national_cancer_data_repository/default.aspx

19. Charlson ME, Pompei P, Ales KL, et al. A new method of classifying prognostic comorbidity in longitudinal studies: development and validation. J Chronic Dis 1987;40:373-83.

20. Berglund A, Garmo H, Tishelman C, et al. Comorbidity, treatment and mortality: a population based cohort study of prostate cancer in PCBaSe Sweden. J Urol 2011;185:833-9.

21. Janssen-Heijnen ML, Smulders S, Lemmens VE, et al. Effect of comorbidity on the treatment and prognosis of elderly patients with non-small cell lung cancer. Thorax 2004;59:602-7.

22. Department of Communities and Local Government. Indices of Deprivation 2007. http://www.communities.gov.uk/publications/ communities/indicesdeprivation 07 .

23. White IR, Royston P, Wood AM. Multiple imputation using chained equations: issues and guidance for practice. Stat Med 2011;30:377-99.

24. Royston P, Parmar MK. Flexible parametric proportional-hazards and proportional-odds models for censored survival data, with application to prognostic modelling and estimation of treatment effects. Stat Med 2002;21:2175-97.

25. Lambert PC, Royston P. Further development of flexible parametric models for survival analysis. Stata J 2009;9:265-90.

26. Team R.D.C. R: A Language and Environment for Statistical Computing Vienna Austria: R Foundation for Statistical Computing, 2008.

27. Asmis TR, Ding K, Seymour L, et al. Age and comorbidity as independent prognostic factors in the treatment of non small-cell lung cancer: a review of National Cancer Institute of Canada Clinical Trials Group trials. J Clin Oncol 2008;26:54-9.

28. Travis WD. Reporting lung cancer pathology specimens. Impact of the anticipated 7th Edition TNM classification based on recommendations of the IASLC Staging Committee. Histopathology 2009;54:3-11.

29. Smith GD, Hart C, Watt G, et al. Individual social class, area-based deprivation, cardiovascular disease risk factors, and mortality: the Renfrew and Paisley Study. J Epidemiol Community Health 1998;52:399-405.

30. Smith GD, Hart C. Re: "Use of census based aggregate variables to proxy for socioeconomic group: evidence from national samples". Am $J$ Epidemiol 1999;150:996-7. 
31. Louwman WJ, Aarts MJ, Houterman S, et al. A 50\% higher prevalence of life-shortening chronic conditions among cancer patients with low socioeconomic status. $\mathrm{Br} \mathrm{J}$ Cancer 2010;103:1742-8.

32. Dalton SO, Frederiksen BL, Jacobsen E, et al. Socioeconomic position, stage of lung cancer and time between referral and diagnosis in Denmark, 2001-2008. Br J Cancer 2011;105:1042-8.

33. Halpern MT, Ward EM, Pavluck AL, et al. Association of insurance status and ethnicity with cancer stage at diagnosis for 12 cancer sites: a restrospective analysis. Lancet Oncol 2008;9:222-31.

34. Riaz SP, Luchtenborg $\mathrm{M}$, Jack $\mathrm{RH}$, et al. Variation in surgical resection for lung cancer in relation to survival: populationbased study in England 2004-2006. Eur J Cancer 2011;24:24.

35. Patel N, Adaita R, Mellemgaard A, et al. Variation in the use of chemotherapy in lung cancer. Br J Cancer 2007;96:886-90.
36. Bungay $\mathrm{H}$. Cancer and health policy: the postcode lottery of care. Soc Pol Admin 2005;39:35-48.

37. Crawford SM, Sauerzapf V, Haynes R, et al. Social and geographical factors affecting access to treatment of lung cancer. $\mathrm{Br} \mathrm{J}$ Cancer 2009;101:897-901

38. Kogevinas M, Pearce N, Susser M, et al. Social Inequalities and Cancer. Vol. 138. Lyon: IARC Scientific Publications, International Agency for Research on Cancer, 1997.

39. Brewster DH, Thomson CS, Hole DJ, et al. Relation between socioeconomic status and tumour stage in patients with breast, colorectal, ovarian, and lung cancer: results from four national, population based studies. BMJ 2001; 322:830-1.

40. Feinstein AR, Sosin DM, Wells CK. The Will Rogers phenomenon Stage migration and new diagnostic techniques as a source of misleading statistics for survival in cancer. $N$ Engl J Med 1985;312:1604-8. 